\title{
Daminozide, Root Pruning, Trunk Scoring, and Trunk Ringing Effects on Fruit Ripening and Storage Behavior of 'McIntosh' Apple
}

\author{
D.C. Elfving \\ Horticultural Research Institute of Ontario, Box 587 Simcoe, Ont. N3Y 4N5, Canada \\ E.C. Lougheed ${ }^{2}$ \\ Department of Horticultural Sciences, University of Guelph, Guelph, Ont. N1G 2W1, Canada
}

R.A. Cline ${ }^{1}$

Horticultural Research Institute of Ontario, Vineland Station, Ont. LOR 2EO, Canada

Additional index words. Malus domestics, Alar, M.7 rootstock

\begin{abstract}
A midsummer foliar daminozide (DZ) application (750 mg a.i./liter) to 'Macspur McIntosh'/M.7 apple trees (Malus domestics Borkh.) reduced preharvest drop and retarded flesh firmness loss and starch hydrolysis when tested at harvest; DZ also reduced fruit ethylene production at harvest and after 19 weeks of storage at $0.5 \mathrm{C}$. Root pruning at full bloom (May) resulted in increased soluble solids concentration (SSC) and firmer flesh and less starch hydrolysis at harvest, but not consistently each year. Full-bloom root pruning reduced the incidence of stem-cavity browning and brown core, but again not each year. Full-bloom root pruning did not influence ethylene evolution at harvest but did reduce post-storage ethylene evolution in two of three seasons. Full-bloom root pruning generally was less effective than DZ in altering fruit behavior, while root pruning later than full bloom had virtually no effect. Trunk scoring or ringing increased SSC and retarded loss of flesh firmness before harvest and following storage, but had little effect on starch hydrolysis. Scoring or ringing decreased incidence of some disorders and reduced poststorage ethylene evolution, although these treatments had little effect on ethylene production at harvest. Trunk scoring influenced some fruit characteristics more strongly than DZ. Fruit size was not affected by any treatment in any year. Chemical name used: butanedioic acid mono (2,2 -dimethylhydrazide) (daminozide).
\end{abstract}

Midsummer daminozide (DZ, Uniroyal Chemical, Middlebury, Corm.) sprays have been widely used on 'McIntosh' apple for control of preharvest drop, fruit maturation, and problems of quality deterioration during and after storage (Edgerton and Blanpied, 1970; Forshey, 1970; Greene et al., 1977; Looney, 1975a, 1975b; Miller and Lougheed, 1971; Williams et al., 1964). However, this product was withdrawn from commercial sales channels in Canada and the United States by the manufacturer in June 1989. Elfving et al. (1987) reported that paclobutrazol (PBZ) had similar, effects to DZ on fruit characteristics. However, Elfving et al. (1990) reported that further evaluations did not demonstrate that PBZ or uniconazole could effectively replace DZ on 'McIntosh'.

Root pruning reduced preharvest drop and fruit size, increased fruit flesh firmness and soluble solids content (SSC) at harvest, and altered fruit mineral-nutrient content (Schupp and Ferree, 1987, 1988). Schumacher et al. (1986) reported no change in fruit firmness, although fruit size was reduced when trees were root-pruned at full bloom, but found that root pruning reduced that incidence of bitter-pit (Schumacher et al., 1978).

Scoring and ringing of trunks or branches also influence fruit behavior. Trunk scoring 19 days after full bloom reduced fruit size in 'Cortland' and 'Delicious' apples, reduced fruit firmness in 'Delicious', and increased SSC in both cultivars (Greene and Lord, 1978). After storage, 'Cortland' apples from scored trees

Received for publication 13 July 1990. Mention of a product or trade name does not constitute a guarantee or warranty of the product by the Hort. Res. Inst. of Ontario nor an endorsement over similar products not mentioned. The cost of publishing this paper was defrayed in part by the payment of page charges. Under postal regulations, this paper therefore must be hereby marked advertisement solely to indicate this fact.

'Research Scientist.

'Professor. showed a higher incidence of bitter-pit and cork, while 'Delicious' fruit exhibited more breakdown.

Branch ringing of 'Granny Smith' apple trees 25 or 50 days before harvest increased fruit size and SSC without influencing flesh firmness at harvest (Gatti and Gil, 1982). Fruit from ringed branches had a higher starch content at harvest, suggesting a delay in the onset of fruit maturation. Following 30 days of cold storage, 'Granny Smith' fruit from ringed branches still exhibited a higher starch content, as well as higher SSC and acidity, but flesh firmness was not different from the control. After 15 days at 20C, fruit from ringed branches retained higher SSC and acidity than untreated fruit, although tissue firmness differences had disappeared.

The experiments described here were conducted on cropping 'Macspur McIntosh'/M.7 trees to assess effects of root pruning, trunk scoring, and trunk ringing (girdling) on fruit characteristics at harvest and after air storage and to compare effects of these treatments with those of DZ.

\section{Materials and Methods}

Three field trials were conducted at the Horticultural Experiment Station, Simcoe, Ont., between 1987 and 1989. All trials used single, whole-tree plots in randomized complete-block designs. DZ was applied to runoff with a handgun. Root pruning was carried out with a tractor-mounted shank capable of reaching $40 \mathrm{~cm}$ in depth. Trunks were scored or ringed using a Juran manual girdling tool (Juran Metal Works, Rishon Le Zion, Israel) modified to make a single cut through the bark to the xylem. A 2-mm-wide strip of bark was removed on ringed trees. Postharvest and poststorage fruit characteristics were evaluated at the Dept. of Horticultural Sciences (DHS), Univ. of Guelph. Counted and weighed fruit samples of 12 to $15 \mathrm{~kg}$ from each replicate tree were sent to the DHS immediately after harvest. Subsamples from each replicate were used for harvest evalua- 
tions. The remaining fruit were stored in air at $0.5 \mathrm{C}$ and $90 \% \%$ to $95 \% \mathrm{RH}$ for 19 weeks before post-storage evaluations.

Daminozide vs. root and trunk modification, one date (Expt. 1 ; 1987). Each of the following six treatments was applied to a separate set of seven replicate, 7-year-old trees: 1) control (no treatment); 2) DZ (750 mg a.i/liter) on 7 July; 3) root pruning along the row $80 \mathrm{~cm}$ from the trunk on each side of each tree at $40 \mathrm{~cm}$ depth at full bloom (8 May); 4) trunk scoring with a single, circumferential cut above the lowest scaffold limb on 11 June; 5) trunk ringing (removal of a 2-mm ring of bark) above the lowest scaffold limb on 11 June; 6) trunk scoring, as described, above the lowest scaffold limb on 30 July. The trees were planted at $4.3 \times 6.7 \mathrm{~m}$ on a well-drained Bookton soil consisting of 40 to $100 \mathrm{~cm}$ of sandy sediments over glaciolacustrine clays and had been cropping for 4 years (Hohner and Presant, 1989). Apple samples were harvested from each tree on 1 and 8 Sept. Fruit were sampled from above the score or ring on trees receiving those treatments.

All fruit on each tree and all drops from each tree were counted and weighed following harvest of the second fruit sample. Fruit counts on the limb below the score or ring in August and counts and weights at harvest permitted assessment of drop as a percentage of the crop above the score or ring.

Daminozide vs. root and trunk modification, several dates (Expt. 2; 1988). Each of the following 10 treatments was applied to a separate set of 10 replicate, 7 -year-old trees: 1) control (no treatment); 2) DZ (750 mg a.i/liter) on 28 June; 3) root pruning along the row $80 \mathrm{~cm}$ from the trunk on each side of each tree at a depth of $40 \mathrm{pm}$ at one of each of the following times: a) full bloom (18 May), b) early summer (15 June), c) midsummer (15 July), or d) late summer (15 Aug.); 4a-d trunk scoring with a single, circumferential cut below the lowest scaffold limb at each of the times when root pruning was done. Trees were planted at $4.3 \times 6.7 \mathrm{~m}$ on an intermediate to welldrained Watford-Maplewood soil complex of mixed sandy and loamy sediments over glaciolacustrine clays and had been cropping for 4 years (Hohner and Presant, 1989). Apple samples were harvested from each tree on 8 and 15 Sept. All harvested fruit and all drops from each tree were counted and weighed following harvest of the second fruit sample;

On the first fruit sampling date, 20 additional apples were collected from each test tree for flesh mineral-nutrient analyses. A composite subsample of fruit flesh from the 20 fruits was wet-ashed at 550C for analysis according to the methods of Bodnar et al. (1983).

DZ vs. type and time of root and trunk modification (Expt. 3 ; 1989). Each of the following eight treatments was applied to a separate set of six replicate, 9-year-old trees planted at $4.3 \mathrm{x}$ $6.7 \mathrm{~m}$ on a well-drained Bookton soil (Hohner and Presant, 1989): 1) control (no treatment); 2) DZ (750 mg a.i./liter) on 6 July; 3) root pruning along the row $80 \mathrm{~cm}$ from the trunk on each side of each tree at full bloom (23 May) at either a) 20 or b) $40 \mathrm{~cm}$ depth; 4a,b root pruning as described above on 1 Aug.; 5) trunk scoring below the lowest scaffold limb on 22 June; 6) two scoring cuts, each around an opposite half of the trunk on 22 June. The ends of the two half-scoring cuts were vertically separated by $\approx 3 \mathrm{~cm}$ to leave a band of intact bark between the top and roots of the trees. These trees had been cropping for 6 years. Apple samples were harvested from each tree on 12 and 19 Sept. All harvested fruit and all drops from each tree were counted and weighed following harvest of the second fruit sample.

Fruit flesh firmness was measured on pared flesh on opposite sectors of each of 10 apples per replicate with an Effegi penetrometer (Effegi, Alfonsine, Italy) using an 11. 1-mm tip. Juice expressed from the apples during the firmness measurements was collected, and SSC was assessed on the composite juice sample with an Atago model N1 hand refractometer (American Optical, Buffalo, N.Y.). Fruit maturity was assessed on the same 10 apples using the starch-iodine test and rating starch patterns against a 'McIntosh' standard (Phillips and Poapst, 1952; Poapst et al., 1959; Priest and Lougheed, 1981; Smith et al., 1979). Following storage, 10 apples per replicate were assessed for tissue firmness and SSC. Disorders were rated visually on 25 apples per replicate after storage plus 1 week at room temperature $(\approx 20 \mathrm{C})$ on a scale from $\mathrm{O}$ (no symptoms) to 3 (severe symptoms),

At harvest and following storage, 10 apples per replicate were placed in a flow-through system to measure ethylene production at 20C (lougheed and Franklin, 1968). The effluent air was automatically sampled and 3-ml samples injected into a Beckman GC-5 gas chromatography (Beckman Instruments, Fullerton, Calif.) with dual flame ionization and thermal conductivity detectors (Lougheed et al., 1969). Ethylene production was calculated from the concentration in the effluent airstream.

All percentage values were transformed using the arcsin of the square root before analysis. Analyses of variance were performed using the General Linear Models (GLM) procedure of the Statistical Analysis System (SAS) program package (SAS Institute, Cary, N.C.). Where a significant F test was observed, means were separated using Duncan's multiple-range test.

\section{Results}

There were no significant interactions between treatments and harvest sampling dates in any experiment.

Expt. 1. Preharvest drop and sample fruit weight were not influenced by any treatment (Table 1). Fruit from the DZ and all trunk or root treatments had firmer flesh than controls at harvest, but only trunk ringing resulted in firmer flesh than that produced by DZ. All trunk injury treatments increased SSC relative to the control or DZ, but only trunk ringing increased SSC above that produced by root pruning at full bloom (8 May). DZ, root pruning, and July trunk scoring retarded starch hydrolysis compared to controls, but DZ was more effective than all other treatments except July trunk scoring. However, ethylene evolution at harvest was lower than control only for DZ and July trunk scoring. Differences between harvest dates reflected changes due to continued fruit growth and the onset of ripening.

Following 19 weeks of storage, fruit from trees root-pruned at full bloom (8 May) or scored or ringed in June had firmer flesh than control or DZ-treated fruit after 1 day at 20C (Table $2)$. By 1 week after storage, fruit from the root-pruning and June scoring and ringing treatments still had firmer flesh than untreated fruit, and ringing produced firmer flesh than any other treatment. SSC was higher for all trunk injury treatments than for root pruning, DZ, or control. Moderate to severe scald was increased by DZ and reduced by June scoring. Root pruning and July scoring decreased the incidence of stem-cavity browning, while root pruning or any trunk injury treatment reduced the incidence of brown core compared to DZ or control fruit. Internal breakdown was uninfluenced by any treatment. Ethylene evolution 2 days after storage was lower for all treatments than for the control, with DZ and ringing leading to the least ethylene production. Where harvest-date effects were significant, they were primarily related to maturity of the fruit at har- 
Table 1. Effects of daminozide (DZ), root pruning (RP), trunk scoring (SC), or trunk ringing

(RG) in 1987 on 'McIntosh' fruit at harvest in 1987 (Expt. 1).

\begin{tabular}{|c|c|c|c|c|c|c|}
\hline Factor & $\begin{array}{c}\text { Preharvest } \\
\text { drop } \\
\text { (\% of yield) }\end{array}$ & $\begin{array}{c}\text { Fruit } \\
\text { wt } \\
(\mathrm{g})\end{array}$ & $\begin{array}{c}\text { Flesh } \\
\text { firmness } \\
(\mathrm{N})\end{array}$ & $\begin{array}{c}\text { Soluble } \\
\text { solids } \\
\text { concn } \\
(\%) \\
\end{array}$ & $\begin{array}{l}\text { Starch } \\
\text { index }^{2} \\
\end{array}$ & $\begin{array}{c}\mathrm{C}_{2} \mathrm{H}_{4} \\
\left(\mu \mathrm{kg} \mathrm{kg}^{-1} \cdot \mathrm{hr}^{-1}\right) \\
\end{array}$ \\
\hline \multicolumn{7}{|c|}{ Treatment and date } \\
\hline Control & $13 \mathrm{a}$ & $160 a$ & $67.6 \mathrm{~d}$ & $12.0 \mathrm{c}$ & $4.0 \mathrm{a}$ & $15.4 \mathrm{a}$ \\
\hline DZ 7 July & $8 \mathrm{a}$ & $160 \mathrm{a}$ & $71.2 \mathrm{bc}$ & $12.2 \mathrm{c}$ & $3.3 \mathrm{~d}$ & $1.6 \mathrm{c}$ \\
\hline RP 8 May & $10 \mathrm{a}$ & $160 a$ & $69.8 \mathrm{c}$ & $12.4 \mathrm{bc}$ & $3.7 \mathrm{bc}$ & $12.7 \mathrm{ab}$ \\
\hline SC 11 June & $9 \mathrm{a}$ & $160 \mathrm{a}$ & $72.0 \mathrm{ab}$ & $12.7 \mathrm{ab}$ & $4.0 a b$ & $7.9 \mathrm{abc}$ \\
\hline RG 11 June & $8 \mathrm{a}$ & $160 \mathrm{a}$ & $.73 .2 \mathrm{a}$ & $13.1 \mathrm{a}$ & $3.8 \mathrm{abc}$ & $7.9 \mathrm{abc}$ \\
\hline SC 30 July & $11 \mathrm{a}$ & $160 \mathrm{a}$ & $70.2 \mathrm{c}$ & $12.8 \mathrm{ab}$ & $3.5 \mathrm{~cd}$ & $4.5 \mathrm{bc}$ \\
\hline \multicolumn{7}{|l|}{ Harvest datex } \\
\hline 1 Sept. & -- & $150 \mathrm{~b}$ & $71.8 \mathrm{a}$ & $12.5 \mathrm{a}$ & $3.2 \mathrm{~b}$ & $3.1 \mathrm{~b}$ \\
\hline 8 Sept. & -- & $170 \mathrm{a}$ & $69.5 \mathrm{~b}$ & $12.6 \mathrm{a}$ & $4.3 \mathrm{a}$ & $13.0 \mathrm{a}$ \\
\hline
\end{tabular}

${ }^{2}$ Mean treatment rating on a scale from 1 to 9 (immature to overmature).

'DZ applied at $750 \mathrm{mg}$ a.i./liter. Mean separation within columns by Duncan's multiple range test $(\mathrm{P}=0.05)$. Each preharvest-drop mean contains seven observations. All other means contain 14 observations each.

${ }^{x}$ Mean separation within columns by $\mathrm{F}$ test $(\mathrm{P}=0.05)$. Each mean contains 42 observations.

Table 2. Effects of daminozide, (DZ), root pruning (RP), trunk scoring (SC), or ringing (RG) in 1987 on 'McIntosh' fruit-quality characteristics following 19 weeks in air at $0.5 \mathrm{C}$ (Expt. 1).

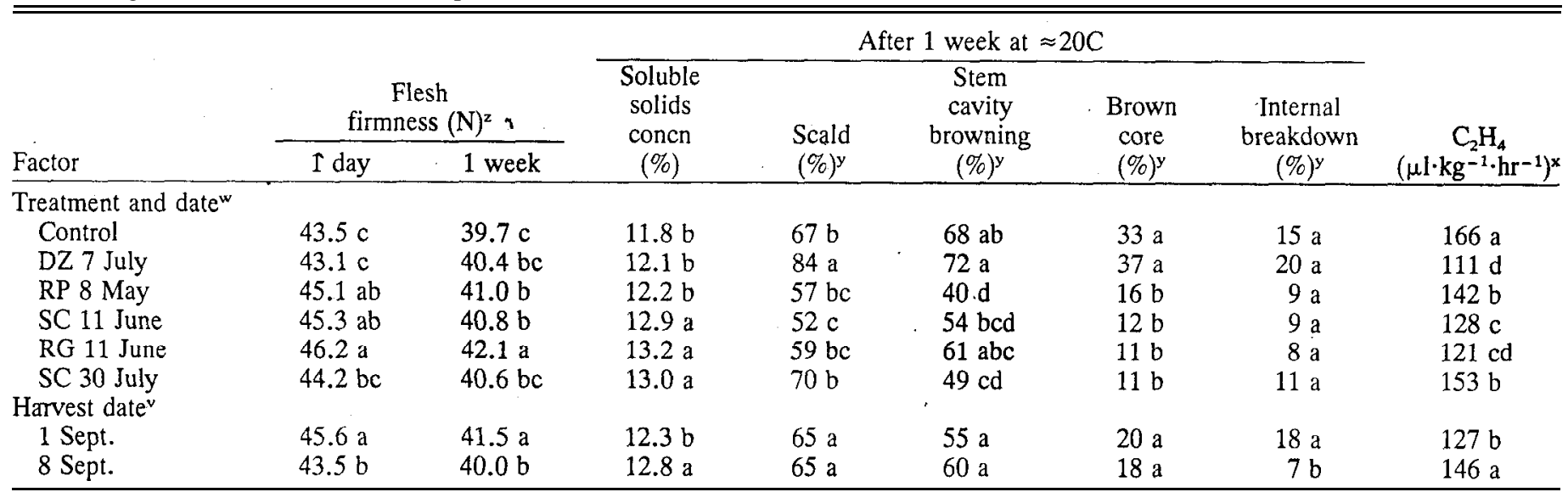

${ }^{2}$ Evaluation after 1 day or 1 week at room temperature ( 20C).

'Percentage of 25 -fruit samples showing moderate (rating of 2) to severe (rating of 3) symptoms.

${ }^{*}$ After 2 days at $20 \mathrm{C}$.

"DZ applied at $750 \mathrm{mg}$ a.i./liter. Mean separation within columns by Duncan's multiple range test $(\mathrm{P}=0.05)$. Each mean contains 14 observations.

'Mean separation within columns by $\mathrm{F}$ test $(\mathrm{P}=0.05)$. Each mean contains 42 observations.

vest, although the higher incidence of internal breakdown at the earlier harvest date was not expected.

Expt. 2. Fruit flesh $\mathrm{K}$ content at harvest was uninfluenced by any treatment (Table 3). Compared to controls, flesh $\mathrm{Ca}$ was reduced by root pruning and scoring in July, but flesh $\mathrm{Mg}$ was reduced only by June scoring. DZ and July scoring decreased preharvest drop compared to the control. There was little effect of any treatment on sample fruit weight, although full-bloom scoring (18 May) produced smaller apples than July root pruning or either August scoring or root pruning. Flesh firmness at harvest was greater for DZ than for the control, and greater yet for trunk scoring in May, June, or July. SSC was increased by June root pruning and by scoring treatments in June or later. Only DZ delayed starch hydrolysis, but ethylene evolution at harvest was not influenced by any treatment. Changes due to sample harvest date were as expected.
Following 19 weeks of storage plus 1 day at 20C, fruit from trees scored 18 May were firmer than the rest. After 1 week at 20C, firmness was equal for those from trees scored 18 Mayor 15 July and firmer than fruit from the other treatments, except for those root pruned 15 June or scored 15 June or 15 Aug. (Table 4). SSC after storage was higher than for the control for June root pruning and both full-bloom and August scoring, higher yet for June scoring, and highest for July scoring. The incidence of moderate to severe storage scald was significantly reduced by scoring in May, June, or July. Stem-cavity browning was similarly reduced by full bloom or July scoring. The incidence of brown core was significantly higher than for the control in DZ-treated fruit, and significantly lower in fruit from trees scored in May, June, or July. Incidence of internal breakdown was generally low, but least in control and full-bloom scoring treatments. Ethylene evolution after 1 day at 20C was lower than 
Table 3. Effects of daminozide (DZ), root pruning (RP), or trunk scoring (SC) in 1988 on 'McIntosh' fruit at harvest in 1988 (Expt. 2)

\begin{tabular}{|c|c|c|c|c|c|c|c|c|c|}
\hline \multirow[b]{2}{*}{ Factor } & \multicolumn{3}{|c|}{$\begin{array}{c}\text { Fruit flesh } \\
\text { content } \\
\text { (mg/100 g f. wt) }\end{array}$} & \multirow{2}{*}{$\begin{array}{c}\text { Preharvest } \\
\text { drop } \\
\text { (\% of yield) }\end{array}$} & \multirow{2}{*}{$\begin{array}{c}\text { Fruit } \\
\text { wt } \\
\text { (g) } \\
\end{array}$} & \multirow{2}{*}{$\begin{array}{c}\text { Flesh } \\
\text { firmness } \\
(\mathrm{N})\end{array}$} & \multirow{2}{*}{$\begin{array}{l}\text { Soluble } \\
\text { solids } \\
\text { concn } \\
(\%)\end{array}$} & \multirow{2}{*}{$\begin{array}{l}\text { Starch } \\
\text { inde } x^{z}\end{array}$} & \multirow{2}{*}{$\begin{array}{c}\mathrm{C}_{2} \mathrm{H}_{4} \\
\left(\mu \mathrm{l} \cdot \mathrm{kg}^{-1} \cdot \mathrm{hr}^{-1}\right)\end{array}$} \\
\hline & $\mathrm{K}$ & $\mathrm{Ca}$ & $\mathrm{Mg}$ & & & & & & \\
\hline \multicolumn{10}{|c|}{ Treatment and date } \\
\hline Control & $139 \mathrm{a}$ & $3.9 \mathrm{ab}$ & $4.4 \mathrm{a}$ & $12 \mathrm{a}$ & $130 \mathrm{ab}$ & $63.4 \mathrm{~d}$ & $12.4 \mathrm{e}$ & $3.6 \mathrm{a}$ & $7.9 \mathrm{a}$ \\
\hline DZ 28 June & $116 \mathrm{a}$ & $3.8 \mathrm{bc}$ & $4.3 \mathrm{a}$ & $5 c$ & $130 \mathrm{ab}$ & $66.8 \mathrm{~b}$ & $12.3 \mathrm{e}$ & $3.2 \mathrm{~b}$ & $0.9 \mathrm{a}$ \\
\hline RP 18 May & 83 a & $3.8 \mathrm{bc}$ & $3.9 \mathrm{ab}$ & $9 a b$ & $130 \mathrm{ab}$ & $64.7 \mathrm{~cd}$ & $12.7 \mathrm{de}$ & $3.5 \mathrm{a}$ & $3.4 \mathrm{a}$ \\
\hline RP 15 June & $103 a$ & $3.8 \mathrm{bc}$ & $4.2 \mathrm{a}$ & $10 a b$ & $130 \mathrm{ab}$ & $64.6 \mathrm{~cd}$ & $13.0 \mathrm{~cd}$ & $3.4 \mathrm{ab}$ & $3.3 \mathrm{a}$ \\
\hline RP 15 July & $71 \mathrm{a}$ & $3.6 \mathrm{c}$ & $4.0 \mathrm{ab}$ & $8 \mathrm{abc}$ & $140 \mathrm{a}$ & $64.1 \mathrm{~cd}$ & $12.7 \mathrm{de}$ & $3.4 \mathrm{ab}$ & $2.2 \mathrm{a}$ \\
\hline RP 15 Aug. & $113 a$ & $3.9 \mathrm{ab}$ & $4.1 \mathrm{a}$ & $8 \mathrm{abc}$ & $140 \mathrm{a}$ & $65.4 b c$ & $12.6 \mathrm{e}$ & $3.6 \mathrm{a}$ & $4.4 \mathrm{a}$ \\
\hline SC 18 May & 92 a & $4.4 \mathrm{a}$ & $3.9 \mathrm{ab}$ & $10 a b$ & $120 \mathrm{~b}$ & $68.7 \mathrm{a}$ & $12.7 \mathrm{de}$ & $3.5 \mathrm{a}$ & $3.8 \mathrm{a}$ \\
\hline SC 15 June & $76 a$ & $4.1 \mathrm{ab}$ & $3.5 \mathrm{~b}$ & $9 \mathrm{ab}$ & $130 \mathrm{ab}$ & $68.3 \mathrm{a}$ & $13.4 \mathrm{~b}$ & $3.5 \mathrm{a}$ & $0.9 \mathrm{a}$ \\
\hline SC 15 July & $97 \mathrm{a}$ & $3.4 \mathrm{c}$ & $4.0 \mathrm{ab}$ & $6 \mathrm{bc}$ & $130 \mathrm{ab}$ & $68.4 \mathrm{a}$ & $14.1 \mathrm{a}$ & $3.4 a b$ & $2.7 \mathrm{a}$ \\
\hline SC 15 Aug. & $120 \mathrm{a}$ & $3.8 \mathrm{bc}$ & $4.4 \mathrm{a}$ & $8 a b c$ & $140 \mathrm{a}$ & $66.6 \mathrm{~b}$ & $13.3 \mathrm{bc}$ & $3.3 \mathrm{ab}$ & $1.7 \mathrm{a}$ \\
\hline \multicolumn{10}{|l|}{ Harvest date $\mathrm{e}^{\mathrm{x}}$} \\
\hline 8 Sept. & -- & --- & --- & --- & $130 \mathrm{~b}$ & $67.8 \mathrm{a}$ & $12.5 \mathrm{~b}$ & $3.0 \mathrm{~b}$ & $1.6 \mathrm{~b}$ \\
\hline 15 Sept. & --- & --- & --- & --- & $140 \mathrm{a}$ & $64.4 \mathrm{~b}$ & $13.3 \mathrm{a}$ & $3.8 \mathrm{a}$ & $4.6 \mathrm{a}$ \\
\hline
\end{tabular}

${ }^{2}$ Mean treatment rating on a scale from 1 to 9 (immature to overmature).

'DZ applied at $750 \mathrm{mg}$ a.i./liter. Mean separation within columns by Duncan's multiple range test $(P=0.05)$. Means for flesh nutrients and preharvest drop contain six observations each; all other means contain 12 observations each.

'Mean separation within columns by $\mathrm{F}$ test $(P=0.05)$. Each mean contains 60 observations.

Table 4. Effects of daminozide (DZ), root pruning (RP), trunk scoring (SC), or ringing (RG) in 1988 on 'McIntosh' fruit-quality characteristics after 19 weeks in air at $0.5 \mathrm{C}$ (Expt. 2).

\begin{tabular}{|c|c|c|c|c|c|c|c|c|}
\hline \multirow[b]{3}{*}{ Factor } & \multirow{2}{*}{\multicolumn{2}{|c|}{$r$}} & \multicolumn{5}{|c|}{ After 1 week at $\approx 20 \mathrm{C}$} & \multirow{3}{*}{$\begin{array}{c}\mathrm{C}_{2} \mathrm{H}_{4} \\
\left(\mu \mathrm{l} \cdot \mathrm{kg}^{-1} \cdot \mathrm{hr}^{-1}\right)^{\mathbf{x}} \\
\end{array}$} \\
\hline & & & \multirow{2}{*}{$\begin{array}{c}\text { Soluble } \\
\text { solids } \\
\text { concn } \\
(\%) \\
\end{array}$} & \multirow{2}{*}{$\begin{array}{l}\text { Scald } \\
(\%)^{y}\end{array}$} & \multirow{2}{*}{$\begin{array}{c}\text { Stem } \\
\text { cavity } \\
\text { browning } \\
(\%)^{y}\end{array}$} & \multirow{2}{*}{$\begin{array}{l}\text { Brown } \\
\text { core } \\
(\%)^{y}\end{array}$} & \multirow{2}{*}{$\begin{array}{c}\text { Internal } \\
\text { breakdown } \\
(\%)^{y}\end{array}$} & \\
\hline & 1 day & 1 week & & & & & & \\
\hline \multicolumn{9}{|c|}{ Treatment and datew } \\
\hline Control & $41.4 \mathrm{~b}$ & $40.2 \mathrm{~b}$ & $12.8 \mathrm{~d}$ & $36 \mathrm{ab}$ & $26 a$ & $19 \mathrm{~b}$ & $1 \mathrm{bc}$ & $144 \mathrm{ab}$ \\
\hline DZ 28 June & $41.2 \mathrm{~b}$ & $40.4 \mathrm{~b}$ & $12.7 \mathrm{~d}$ & $32 \mathrm{abc}$ & 29 a & $35 \mathrm{a}$ & $5 \mathrm{a}$ & $113 \mathrm{~d}$ \\
\hline RP 18 May & $40.5 \mathrm{~b}$ & $40.1 \mathrm{~b}$ & $12.9 \mathrm{~d}$ & $32 \mathrm{abc}$ & $26 a$ & $18 \mathrm{~b}$ & $4 a b$ & $147 \mathrm{a}$ \\
\hline RP 15 June & $41.3 \mathrm{~b}$ & $40.9 \mathrm{ab}$ & $13.4 \mathrm{c}$ & $37 \mathrm{ab}$ & $26 a$ & $16 \mathrm{~b}$ & $2 \mathrm{ab}$ & $144 a b$ \\
\hline RP 15 July & $41.4 \mathrm{~b}$ & $40.3 \mathrm{~b}$ & $13.0 \mathrm{~d}$ & $40 \mathrm{a}$ & $29 \mathrm{a}$ & $20 \mathrm{~b}$ & $4 \mathrm{ab}$ & $142 \mathrm{ab}$ \\
\hline RP 15 Aug. & $41.3 \mathrm{~b}$ & $39.9 \mathrm{~b}$ & $12.9 \mathrm{~d}$ & $33 a b c$ & $29 \mathrm{a}$ & $20 \mathrm{~b}$ & $2 a b$ & $144 a b$ \\
\hline SC 18 May & $43.5 \mathrm{a}$ & $41.8 \mathrm{a}$ & $13.5 \mathrm{c}$ & $22 \mathrm{c}$ & $11 \mathrm{c}$ & $4 \mathrm{c}$ & $0 \mathrm{c}$ & $135 \mathrm{bc}$ \\
\hline SC 15 June & $41.3 \mathrm{~b}$ & $40.8 \mathrm{ab}$ & $14.2 \mathrm{~b}$ & $22 \mathrm{c}$ & $22 \mathrm{a}$ & $1 \mathrm{c}$ & $4 \mathrm{ab}$ & $116 \mathrm{~d}$ \\
\hline SC 15 July & $41.6 \mathrm{~b}$ & $41.6 \mathrm{a}$ & $14.7 \mathrm{a}$ & $15 \mathrm{c}$ & $13 \mathrm{bc}$ & $1 \mathrm{c}$ & $5 \mathrm{a}$ & $110 \mathrm{~d}$ \\
\hline SC 15 Aug. & $41.5 \mathrm{~b}$ & $41.0 \mathrm{ab}$ & $13.7 \mathrm{c}$ & $26 b c$ & $21 \mathrm{ab}$ & $14 \mathrm{~b}$ & $4 a b$ & $130 \mathrm{c}$ \\
\hline \multicolumn{9}{|l|}{ Harvest date } \\
\hline 8 Sept. & $41.8 \mathrm{a}$ & $41.1 \mathrm{a}$ & $13.1 \mathrm{~b}$ & $41 \mathrm{a}$ & $26 a$ & $9 \mathrm{~b}$ & $0 \mathrm{~b}$ & $126 \mathrm{~b}$ \\
\hline 15 Sept. & $41.2 \mathrm{~b}$ & $40.3 \mathrm{~b}$ & 13.7 a & $19 \mathrm{~b}$ & $20 \mathrm{~b}$ & $17 \mathrm{a}$ & $8 \mathrm{a}$ & 139 a \\
\hline
\end{tabular}

${ }^{2}$ Evaluation after 1 day or 1 week at room temperature $(\approx 20 \mathrm{C})$.

'Percentage of 25 -fruit samples showing moderate (rating of 2) to severe (rating of 3 ) symptoms.

${ }^{x}$ After 1 day at $20 \mathrm{C}$.

"DZ applied at $750 \mathrm{mg}$ a.i./liter. Mean separation within columns by Duncan's multiple range test $(\mathrm{P}=0.05)$. Each mean contains 12 observations.

'Mean separation within columns by $\mathrm{F}$ test $(\mathrm{P}=0.05)$. Each mean contains 60 observations.

control for DZ and for scoring in June, July, or August. Harvestdate differences reflected the maturity of fruit at harvest and condition at the time of analysis.

Expt. 3. Preharvest drop was significantly lower than for the control and August root pruning for DZ-treated trees and those root-pruned at 20-cm depth at full bloom (23 May; Table 5). Fruit weight was not influenced by any treatment. Flesh was firmer than for the control in DZ-treated fruit and in fruit from both full-bloom (23 May) root-pruning treatments. Both trunkscoring treatments resulted in firmer flesh than DZ application.
SSC was increased over the control or DZ-treated fruit for fullbloom root pruning at the $20-\mathrm{cm}$ depth and by both trunk-scoring treatments. DZ application and full-bloom root pruning at the $20-\mathrm{cm}$ depth reduced starch hydrolysis significantly, as indicated by a low starch index, while DZ and trunk scoring reduced ethylene evolution at harvest.

Following 19 weeks of storage, there were few significant differences in flesh firmness after 1 day or 1 week (Table 6). SSC was highest for both trunk-scoring treatments. The incidence of moderate to severe scald was increased by DZ appli- 
Table 5. Effects of diaminozide (DZ), root pruning (RP), trunk scoring (SC), or scoring each half of the trunk (HSC) in 1989 on 'McIntosh' fruit at harvest in 1989 (Expt. 3).

\begin{tabular}{|c|c|c|c|c|c|c|}
\hline Factor & $\begin{array}{c}\text { Preharvest } \\
\text { drop } \\
\text { (\% of yield) }\end{array}$ & $\begin{array}{c}\text { Fruit } \\
\text { wt } \\
(\mathrm{g})\end{array}$ & $\begin{array}{c}\text { Flesh } \\
\text { firmness } \\
(\mathrm{N})\end{array}$ & $\begin{array}{l}\text { Soluble } \\
\text { solids } \\
\text { concn } \\
(\%)\end{array}$ & $\begin{array}{l}\text { Starch } \\
\text { index }\end{array}$ & $\begin{array}{c}\mathrm{C}_{2} \mathrm{H}_{4} \\
\left(\mu \mathrm{l} \cdot \mathrm{kg}^{-1} \cdot \mathrm{hr}^{-1}\right)\end{array}$ \\
\hline \multicolumn{7}{|c|}{ Treatment, date, and depth $(\mathrm{cm})^{y}$} \\
\hline Control & 30 a & $140 \mathrm{a}$ & $63.8 \mathrm{~d}$ & $11.6 \mathrm{~cd}$ & $4.5 \mathrm{a}$ & $13.6 \mathrm{ab}$ \\
\hline DZ 6 July & $10 \mathrm{~b}$ & 140 a & $66.2 \mathrm{c}$ & $11.5 \mathrm{~d}$ & $3.7 \mathrm{~b}$ & $1.0 \mathrm{~d}$ \\
\hline RP 23 May, 20 & $11 \mathrm{~b}$ & $140 \mathrm{a}$ & $67.0 \mathrm{bc}$ & $12.1 \mathrm{~b}$ & $3.7 \mathrm{~b}$ & 6.2 bcd \\
\hline RP 23 May, 40 & $17 \mathrm{ab}$ & $140 \mathrm{a}$ & $66.2 \mathrm{c}$ & $12.0 \mathrm{bc}$ & $3.9 \mathrm{a}$ & 7.4 bcd \\
\hline RP 1 Aug., 20 & $26 \mathrm{a}$ & 140 a & $63.8 \mathrm{~d}$ & $11.7 \mathrm{bcd}$ & $4.5 \mathrm{a}$ & $18.9 \mathrm{a}$ \\
\hline RP 1 Aug., 40 & $33 \mathrm{a}$ & $140 \mathrm{a}$ & $63.7 \mathrm{~d}$ & $11.7 \mathrm{bcd}$ & $4.4 \mathrm{a}$ & $11.3 \mathrm{abc}$ \\
\hline SC 22 June & $17 a b$ & $140 a$ & $69.6 \mathrm{a}$ & $13.0 \mathrm{a}$ & $4.2 \mathrm{ab}$ & $3.0 \mathrm{~cd}$ \\
\hline HSC 22 June & $22 a b$ & 140 a & $68.5 \mathrm{ab}$ & $12.7 \mathrm{a}$ & $4.2 \mathrm{ab}$ & $7.2 \mathrm{bcd}$ \\
\hline \multicolumn{7}{|l|}{ Harvest date } \\
\hline 12 Sept. & --- & $130 \mathrm{~b}$ & $67.6 \mathrm{a}$. & $11.9 \mathrm{~b}$ & $3.4 \mathrm{~b}$ & $8.3 \mathrm{a}$ \\
\hline 19 Sept. & $-\cdots$ & $140 \mathrm{a}$ & $64.6 \mathrm{~b}$ & $12.1 \mathrm{a}$ & $4.9 \mathrm{a}$ & 9.0 t \\
\hline
\end{tabular}

${ }^{7}$ Mean treatment rating on a scale from 1 to 9 (immature to overmature).

'DZ applied at $750 \mathrm{mg}$ a.i./liter. Mean separation within columns by Duncan's multiple range test $(P=0,05)$.

Each preharvest-drop mean contains six observations; all others contain 12 observations.

${ }^{x}$ Mean separation within columns by $\mathrm{F}$ test $(P=0.05)$. Each mean contains 42 observations.

Table 6. Effects of daminozide (DZ), root pruning (RP), trunk scoring (SC), or scoring each half of the trunk (HSC) in 1989 on 'McIntosh' fruit-quality characteristics after 19 weeks in air at $0.5 \mathrm{C}$ (Expt. 3).

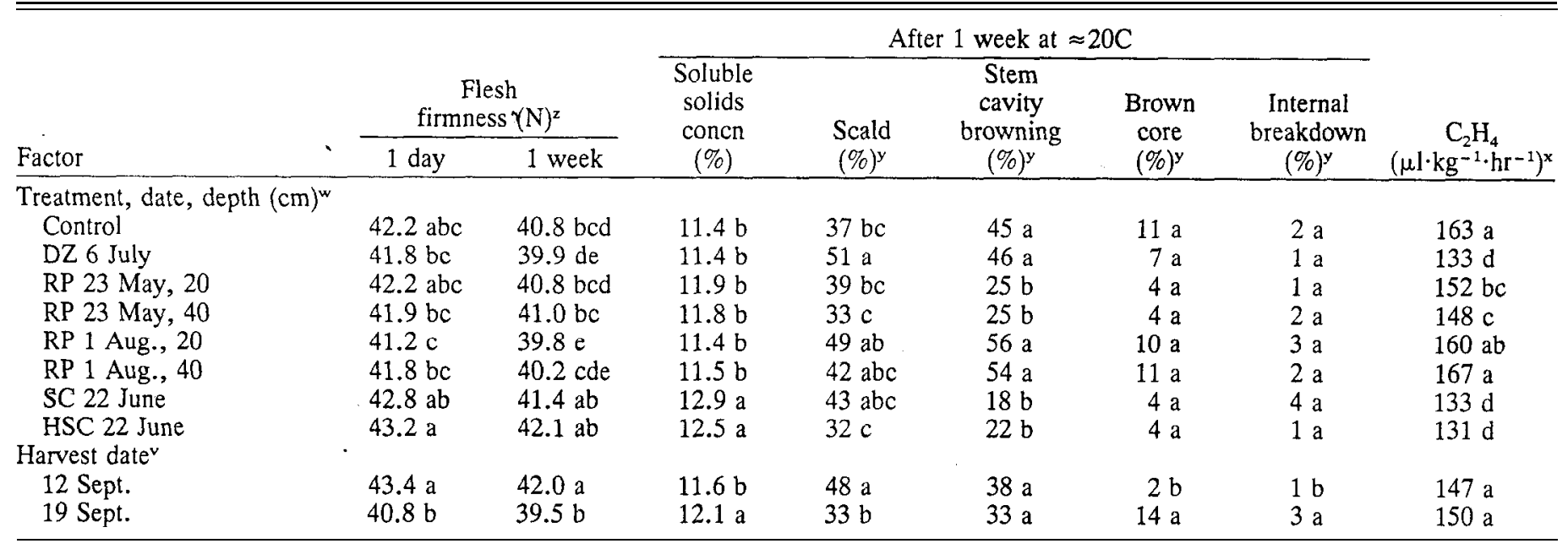

${ }^{2}$ Evaluation after 1 week at room temperature $(\approx 20 \mathrm{C})$.

'Percentage of 25 -fruit samples showing moderate (rating of 2) to severe (rating of 3) symptoms.

${ }^{x}$ After 2 days at $20 \mathrm{C}$.

"DZ applied at $750 \mathrm{mg}$ a.i./liter. Mean separation within columns by Duncan's multiple range test $(P=0.05)$. Each mean contains 12 observations.

Mean separation within columns by $\mathrm{F}$ test $(P=0.05)$. Each mean contains 42 observations.

cation. Compared to other treatments, full-bloom root pruning (23 May) and both trunk-scoring treatments significantly reduced stem-cavity browning. The incidence of brown core and internal breakdown were not influenced by any treatment. Ethylene evolution after 2 days at 20C was significantly lower than for the control for DZ application, full-bloom root pruning, and both trunk-scoring treatments; ethylene evolution for full-bloom root pruning was significantly higher than for DZ application. Harvest-date differences were related to fruit maturity at harvest.

\section{Discussion}

Root pruning at full bloom and summer scoring and ringing of the trunk altered 'McIntosh' fruit behavior at harvest and after 19 weeks of storage at $0.5 \mathrm{C}$. Trunk scoring as late in the growing season as 3 weeks before harvest increased fruit firmness and SSC, confirming the observations of Gatti and Gil (1982). The mechanism(s) inducing these fruit responses to root pruning, trunk scoring, or trunk ringing well before harvest are unclear, but are likely related to changes in assimilate, mineralnutrient, and/or hormonal levels as a result of interruption of the vascular system and subsequent wound-healing activity.

Trunk scoring and ringing sometimes influenced fruit-quality characteristics to a greater extent than DZ. Firmness retention at harvest and after storage was generally better in fruit from scored trees, while occurrence of disorders tended to be reduced. Delayed fruit maturity, as estimated by the starch index, was the only fruit-quality aspect more consistently influenced by DZ than by trunk scoring. The time during the growing 
season when scoring was done had relatively little influence on fruit response to this treatment. Separately scoring each half of the trunk to leave an intact band of bark in 1989 produced effects in fruit virtually identical to those from a complete circumferential scoring cut. Scoring or ringing had little effect on fruit weight in these experiments. Because trunk scoring and -ringing can have significant negative effects on tree and fruit development (Batjer and Westwood, 1963; Greene and Lord, 1983; Toenjes, 1949), considerable caution should be exercised when employing this technique. The effects of repeated trunk scoring over several seasons on tree and fruit responses were not examined in this study.

In the trials reported here, root pruning at full bloom had lessconsistent effects and influenced fewer fruit-quality characteristics than DZ. Root pruning later than full bloom had virtually no effect on fruit at harvest or after storage. Root-pruning did not influence fruit weight. In other studies, root-pruning effects on fruit-quality indices were accompanied by reductions in fruit size (Schumacher et al., 1978; Schupp and Ferree, 1987, 1988). Schupp and Ferree (1988) reported no effect of root-pruning severity on fruit quality of 'Melrose' apples at harvest, but all root-pruning treatments in their study reduced fruit size. The limited effects of root pruning on fruit behavior in the trials reported here may reflect less-severe root pruning than in other studies reporting similar responses (Schumacher et al., 1978; Schupp and Ferree, 1987, 1988). Further research is needed to determine whether fruit-size reduction is consistently associated with root-pruning treatments that may more strongly affect fruit quality characteristics as determined at harvest and after storage.

\section{Literature Cited}

Batjer, L.P. and M.N. Westwood. 1963. Effects of nitrogen, pruning and scoring on growth and bearing characteristics of young Delicious apple trees. Proc. Amer. Soc. Hort. Sci. 82:5-10.

Bodnar, J. M., J.T.A. Proctor, J.E. Laing, and R.A. Cline. 1983. Nutrient changes in apple leaves due to the spotted tentiform leafminer. J. Amer. Soc. Hort. Sci. 108:685-688.

Edgerton, L.J. and G.D. Blanpied. 1970. Interaction of succinic acid 2,2-dimethyl hydrazide, 2-chloroethylphosphonic acid and auxins on maturity, quality and abscission of apples. J. Amer. Soc. Hort. Sci. 95:664-666.

Elfving, D. C., C.L. Chu, E.C. Lougheed, and R.A. Cline. 1987. Effects of daminozide and paclobutrazol treatments on fruit ripening and storage behavior of 'McIntosh' apple. J. Amer. Soc. Hort. Sci. 112:910-915.

Elfving, D. C., E.C. Lougheed, C.L. Chu, and R.A. Cline. 1990. Effects of daminozide, paclobutrazol, and uniconazole treatments on 'McIntosh' apples at harvest and following storage. J. Amer. Soc. Hort. Sci. 115:750-756.

Forshey, C.G. 1970. The use of Alar on vigorous 'McIntosh' apple trees. J. Amer. Soc. Hort. Sci. 95:64-67.

Gatti, R. and G. Gil. 1982. Regulaci6n de la madurez de manzanas cv Granny Smith con ethephon y anilladura. Ciencia e Investig. Agraria 9:27-36.

Greene, D.W. and W.J. Lord. 1978. Evaluation of scoring, limb spreading and growth regulators for increasing flower bud initiation and fruit set on young 'Delicious' apple trees. J. Amer. Soc. Hort. Sci. 103:208-210.

Greene, D.W. and W.J. Lord. 1983. Effects of dormant pruning, summer pruning, scoring and growth regulators on growth, yield, and fruit quality of 'Delicious' and 'Cortland' apple trees. J. Amer. Soc. Hort. Sci. 108:590-595.

Greene, D. W., W.J. Lord, and W.J. Bramlage. 1977. Mid-summer applications of ethephon and daminozide on apples. 1. Effect on 'McIntosh'. J. Amer. Soc. Hort. Sci. 102:491-494.

Hohner, B. and T. Presant. 1989. Soils of the horticultural experiment station, Simcoe. Ont. Inst. of Pedology Publ. 89-3.

Looney, N.E. 1975a. Control of ripening in 'McIntosh' apples. I. Some growth regulator effects on preharvest drop and fruit quality at four harvest dates. J. Amer. Soc. Hort. Sci. 100:330-332.

Looney, N.E. 1975b. Control of ripening in 'McIntosh' apples. II. Effect of growth regulators and $\mathrm{CO}_{2}$ on fruit ripening, storage behavior and shelf life. J. Amer. Soc. Hort. Sci. 100:332-336.

Lougheed, E.C. and E.W. Franklin. 1968. Automated system for measuring respiration period. Can. J. Plant Sci. 48:435-438.

Lougheed, E. C., E.W. Franklin, and R.B. Smith. 1969. Ethylene analyses by automatic gas chromatography. Can. J. Plant Sci. 49:386391.

Miller, S.R. and E.C. Lougheed. 1971. Residual effects of Alar on the carbon dioxide and ethylene production of McIntosh apples at harvest and after long storage. Can. J. Plant Sci. 51:87-91.

Phillips, W.R. and P.A. Poapst. 1952. Storage of apples. Can. Dept. Agr., Hort. Div., Expt. Farms Serv. Publ. 776.

Poapst, P. A., G.M. Ward, and W.R. Phillips. 1959. Maturation of McIntosh apples in relation to starch loss and abscission. Can. J. Plant Sci. 39:257-263.

Priest, K.L. and E.C. Lougheed. 1981. Evaluating apple maturity using the starch-iodine test. Ont. Min. Agr. and Food Factsheet 81-025.

Schumacher, R., F. Fankhauser, and W. Stadler. 1978. Beeinflussung der Fruchtbarkeit und der Fruchtqualität durch den Wurzelschnitt. Schweiz. Z. Obst- und Weinbau 114:56-61.

Schumacher, R., F. Fankhauser, and W. Stadler. 1986. Influence of growth regulators, ringing and root cutting on apple quality and physiological disorders. Acta Hort. 179(2):731-742.

Schupp, J.R. and D.C. Ferree. 1987. Effect of root pruning at different growth stages on growth and fruiting of apple trees. HortScience 22:387-390.

Schupp, J.R. and D.C. Ferree. 1988. Effects of root pruning at four levels of severity on growth and yield of 'Melrose'/M.26 apple trees. J. Amer. Soc. Hort. Sci. 113:194-198.

Smith, R. B., E.C. Lougheed, E.W. Franklin, and 1. McMillan. 1979. The starch iodine test for determining stage of maturation in apples. Can. J. Plant Sci. 59:725-735.

Toenjes, W. 1949. The effect of trunk girdling on inducing earlier bearing of Northern Spy apple trees. Mich. Quart. Bul. 32:23-27.

Williams, M. W., L.P. Batjer, and G.C. Martin. 1964. Effects of Ndimethyl amino succinamic acid (B-Nine) on apple quality. Proc. Amer. Soc. Hort. Sci. 85:17-19. 